\title{
GAG-Ersatztherapie kausalnah und effektiv
}

— Die Glykosaminoglykan-(GAG)-Schicht mit ihrem wesentlichen Bestandteil Chondroitinsulfat bildet als Schutzschicht der Blasenwand die Grenze zwischen Urin und Urothel. Ein Defekt der GAG-Schicht gilt als wahrscheinliche Ursache für die mit chronischen Zystitiden einhergehenden Symptome. Die intravesikale Instillation mit Gepan ${ }^{\circledR}$ instill versorgt die GAG-Schicht mit ihrem Baustein Chondroitinsulfat und behebt den Defekt. Davon können Patienten aller Formen der chronischen Zystitis (interstitielle Zystitis, überaktive Blase, radiogene Zystitis, chronisch-rezidivierende Harnwegsinfekte) profitieren.

Wenn bei der interstitiellen Zystitis (IC) medikamentöse Therapien mit Pentosanpolysulfat, Antihistaminika oder Antidepressiva ausgeschöpft sind, sei die intravesikale Instillationstherapie eine wichtige Option, so Prof. Daniela Schultz-Lampel, Villingen-Schwenningen im Rahmen des Satelliten-Symposiums "Chronische Zystitiden: Ursachen - Diagnose - innovative Therapieoptionen" in Hamburg. „Hier können Erfolgsraten bis zu
$80 \%$ erwartet werden", so Schultz-Lampel. Dies zeigte auch eine multizentrische prospektive Beobachtungsstudie an 286 Patienten mit chronischen Zystitiden [Nordling J, van Ophoven A. 2008]: $77 \%$ der 165 ICPatienten bewerteten die Therapie positiv: Harndrang, Miktionsfrequenz am Tag, Nykturie und Blasenschmerzen besserten sich signifikant.

Rund $25-30 \%$ der onkologischen Patienten, die sich einer Bestrahlung im Beckenbereich unterziehen müssen, entwickeln eine radiogene Zystitis. „Eine große internationale Beobachtungsstudie an 286 Patienten mit chronischen Zystitiden - darunter rund $5 \%$ mit Strahlenzystitis - zeigte, dass die Behandlung mit GAG-Ersatz die Symptome um 25$50 \%$ verbessert", so Dr. Jan-Paul Roovers. Diese Ergebnisse bestätigten sich auch in einer Pilotstudie an 20 Patienten mit radiogener Zystitis [Hazewinkel HH et al. 2011].

Nach Informationen von

Pohl-Boskamp, Hohenlockstedt
G-BA bestätigt Zusatznutzen von Abirateronacetat

Der Gemeinsame Bundesausschuss (G-BA) hat den Beschluss zu Zytiga ${ }^{\oplus}$ (Abirateronacetat) im Rahmen der frühen Nutzenbewertung bekanntgegeben. Darin bestätigt der G-BA den Hinweis auf einen beträchtlichen Zusatznutzen für Zytiga ${ }^{\circledR}$ für solche Patienten, für die eine erneute Therapie mit Docetaxel nicht infrage kommt.

Abirateronacetat wurde in Kombination mit Prednison oder Prednisolon bei Patienten mit metastasiertem kastrationsresistentem Prostatakarzinom (mCRPC) nach Versagen einer Docetaxel-haltigen Chemotherapie im September 2011 von der Europäischen Kommission in einem beschleunigten Verfahren zugelassen.

Die Preisverhandlungen für Zytiga ${ }^{\circledR}$ zwischen dem Spitzenverband Bund der Krankenkassen und Janssen werden Ende September abgeschlossen sein. Das Präparat ist weiterhin erstattungsfähig und kann im Rahmen der Zulassung verschrieben werden.

Nach Informationen von

Janssen-Cilag, Neuss

\section{Testosteronpräparat neu im Sortiment}

Androtop ${ }^{\circledR}$ Gel zur Testosteronsubstitution bei männlichem Hypogonadismus wird seit 1. April 2012 wieder von Dr. Kade/ Besins Pharma GmbH vertrieben. Das GelPräparat stammt aus der Forschung von Besins Healthcare.

Androtop ${ }^{\circledR}$ Gel wird zur effektiven Behandlung des männlichen Hypogonadismus eingesetzt. Es ist individuell dosierbar, trocknet nach dem Auftragen rasch und rückstandslos und gibt nach perkutaner Resorption Testosteron in konstanten Mengen über 24 Stunden in den systemischen Kreislauf ab.

Das Gel erwies sich in klinischen Studien als sehr gut verträglich. Im Unterschied zur Injektionstherapie ist die Behandlung mit Androtop ${ }^{\circledast}$ Gel sehr gut steuerbar. Das $1 \%$ ige hydroalkoholische Testosteron-Gel wird in dosiersicheren, einzeln verpackten Sachets zu $25 \mathrm{mg}$ oder $50 \mathrm{mg}$ Testosteron angeboten.

Nach Informationen von

Dr. Kade/Besins, Berlin
Bei 30-40\% der HWI-Patienten kommt es immer wieder zu Rezidiven, innerhalb von drei Monaten bei fast jedem vierten Betrof-
Nach Informationen von

Bionorica SE, Neumarkt 